\title{
Interatividade Educativa Preservando o Desenvolvimento de Prematuros: Pesquisa Convergente Assistencial
}

\author{
Márcia Helena de Souza Freire ${ }^{1}$, Karoline \\ Petricio Martins ${ }^{2}$ e Ivete Palmira Sanson \\ Zagonel $^{3}$
}

${ }^{1}$ Enfermeira. Professora do Ensino Superior, Doutora em Saúde Pública, Departamento de Enfermagem da Universidade Federal do Paraná, Brasil | marciahelenafreire@gmail.com | https://orcid.org/0000-0003-3941-3673

${ }^{2}$ Enfermeira de UTI neonatal, Mestre em Prática do Cuidado em Saúde, Complexo Hospital de Clínicas, Universidade Federal do Paraná, Brasil | karolinepetricio@gmail.com | https://orcid.org/0000-0003-3599-6584

${ }^{3}$ Enfermeira. Professora do Ensino Superior, Diretora Acadêmica das Faculdades Pequeno Príncipe, Curitiba, Brasil | ivete.zagonel@fpp.edu.br | https://orcid.org/0000-0002-6169-0709

Resumo: Introdução: Na prática assistencial intensiva neonatal com integração dos cuidados à novas tecnologias, é necessária a adequação ambiental e interatividade educativa. Tem-se como questão norteadora: Como promover a reflexão com interatividade, junto à equipe de profissionais de unidade intensiva, com base nas evidências científicas, sobre os cuidados com o desenvolvimento do recémnascido prematuro? Objetivo: descrever parte do processo de uma Pesquisa Convergente Assistencial (PCA), ancorada pela Matriz de Design Instrucional, visando a intervenção nos cuidados aos recém-nascidos pré-termos em Unidade de Terapia Intensiva Neonatal. Método: Pesquisa realizada entre junho e agosto de 2020. Para o cumprimento de uma das etapas da PCA foram desenvolvidas nove oficinas instrucionais. Estas foram organizadas com aulas interativas audiovisuais gravadas por profissionais de saúde da instituição, disponibilizadas por ferramenta Wikiß, na Plataforma Moodle com evidências de producões científicas, perguntas disparadoras, chat e proposta de intervenção. Participaram 68 profissionais que prestam cuidados intensivos ao recém-nascido. Resultados: Os recursos utilizados nas oficinas veicularam aspectos do cuidado ao recém-nascido prematuro, incentivando o (re)conhecimento das repercussões fisiológicas da imaturidade, dentre elas: a imaturidade e as deficiências e comprometimento intelectual e neurológico; consequências das manipulações excessivas; a termorregulação e sobrevivência dos neonatos; contextos de vulnerabilidade familiar e, a importância do acolhimento; os desafios do aleitamento materno ao RNPT; o processo fisiológico do estresse e da dor e, a relevância de um protocolo institucional para alívio. Conclusão: o processo interativo remoto não síncrono, conduzido como parte do desenvolvimento metodológico da Pesquisa Convergente Assistencial, culminou com o estabelecimento participativo de um protocolo institucional inovador para manuseio mínimo do prematuro. Suscitou aos profissionais a releitura de sua prática e, a descoberta de novos conhecimentos favoráveis ao cuidado com o desenvolvimento do recém-nascido prematuro crítico.

Palavras-chave: Recém-nascido Prematuro; Unidades de Terapia Intensiva Neonatal; Enfermagem; Educação Continuada; Tecnologia Educacional.

\section{Educational Interactivity to Preserve Development of Preterm Infant: Converging Care Research}

Abstract: Introduction: New technologies are relevant allies to reduce morbidity and mortality in neonatal care, supported by environmental adequacy and minimization of stimuli, through singular care and behavioral observation. However, integrating care with new technologies, in neonatal intensive care practice, requires educational interactivity. Therefore, the research question was: How to promote reflection with interactivity, with the team of professionals in the intensive care unit, based on scientific evidence, about the care with the development of premature newborns? Objective: to describe a Convergent Care Research, anchored by the Instructional Design Matrix, for intervention in the care of preterm infants in a Neonatal Intensive Care Unit. Method: Convergent Care Research supported by a pedagogical process structured in an Instructional Design Matrix, carried out between June and August 2020. There were nine certified workshops (20h), made available by Wiki ${ }^{\circledR}$ tool, on the Moodle Platform. Participated 68 professionals who provide intensive care to preterm infants, including those who were away from the service, because it was remote. Results: nine instructional workshops were organized for the fulfillment of one of the stages of the research process convergent to care. With interactive audiovisual classes recorded by the institution's health professionals, invited due to their expertise. Included in each workshop, scientific publications, related videos, triggering questions and chat for reflective and purposeful discussion. All resources transmit aspects of care for the premature infants, encouraging the acknowledgment of the physiological repercussions of immaturity, among them: immaturity and deficiencies; intellectual and neurological impairment; consequences of excessive manipulation; the thermoregulation and survival of neonates; contexts of family vulnerability and, the importance of reception; the challenges of breastfeeding to preterm infants; the physiological process of stress and pain; also the relevance of an institutional protocol for relief. Conclusion: the remote nonsynchronous interactive process, methodologically conducted by Convergent Care Research, culminated in the participatory establishment of an innovative institutional protocol for minimal handling of premature infants. It prompted professionals to reread their practice and discover new knowledge favorable to care for the development of critical premature infants.

Keywords: Infant, Premature; Intensive Care Units; Neonatal; Nursing; Education; Continuing; Educational Technology. 


\section{Introdução}

Define-se como recém-nascido pré-termo ou prematuro (RNPT) o nascido anteriormente à $37^{\text {a }}$ semana de gestação (Organização das Nações Unidas [ONU], 2019). Em 2011, foram registrados $9,8 \%$ nascidos prematuros no Brasil. Fato que exige atenção, sobretudo do ponto de vista assistencial, pois há necessidade de planejamento dos cuidados englobando o aspecto desenvolvimental, para a promoção da redução da incidência de morbidades e maior sobrevivência (Marski et al., 2018; Ministério da Saúde [MS], 2020).

Assim, visando a estabilização clínica e sobrevida dos RNPT, estes usualmente são encaminhados, para assistência e tratamento nas Unidades de Terapia Intensiva Neonatal (UTIN). A despeito da relevância inquestionável do serviço ressalta-se a coexistência de elevado risco de danos aos RNPT, devido suas condições de vulnerabilidade e incapacidade de autorregulação, às quais exigem intervenções e procedimentos que favorecem a exposição à dor, estresse, luminosidade e ruídos do ambiente. Fatores que os colocam em situação susceptibilidade elevada ao risco de maturação cerebral anormal e, ao aumento do índice de morbimortalidade (Abdeyazdan et al., 2014; Gaspardo et al., 2010).

A filosofia do cuidado desenvolvimental (Neonatal Individualized Development Care and Assessment Program - NIDCAP®), desenvolvida por Heidelise Als, em 1982, propôs o cuidado para promover um ambiente seguro ao RNPT. De modo que torna possível a identificação das vulnerabilidades do recém-nascido e de seus familiares e, também a intervenção junto aos mesmos, com minimização das possíveis complicações e agravos (Marski et al., 2018).

O NIDCAP® apresenta, como uma de suas bandeiras, o manuseio mínimo do RNPT pela equipe, com evidências científicas de resultados positivos no(s): quadro clínico; padrão de resposta comportamental e fisiológica dos sistemas autonômico, motor e de autorregulação; processos de dor; e, desenvolvimentos neurocomportamental, neurofisiológico e neuroestrutural (Gaspardo et al., 2010). Nessa perspectiva, no Brasil, aliado ao avanço da tecnologia e de protocolos com base na filosofia de cuidados, foi criado o Método Canguru (MC), como política de atenção humanizada a RNPT (Ministério da Saúde [MS], 2020).

Para que sejam difundidas e apreendidas as atualizações e as novas abordagens de cuidado aos RNPT deve ser incentivada a educação permanente da equipe multiprofissional. Para tanto, podem ser utilizados veículos como oficinas e aulas remotas ou à distância (EaD), as quais têm ampliado o acesso aos processos pedagógicos, sobretudo na vigência de impossibilidades presenciais. É incentivado, para este fim, o planejamento e implementação de uma Matriz de Design Instrucional, como um instrumento de gestão do conteúdo a ser abordado pelo objeto de aprendizagem (Costa et al., 2014).

Assim, frente à questão norteadora: Como promover a reflexão remota e com interatividade, junto à equipe de profissionais de unidade intensiva, com base nas evidências científicas sobre os cuidados com o desenvolvimento do recém-nascido prematuro? Esta produção tem como objetivo descrever parte do processo de uma Pesquisa Convergente Assistencial, a qual foi ancorada pela Matriz de Design Instrucional, visando a intervenção nos cuidados aos recém-nascidos pré-termos em Unidade de Terapia Intensiva Neonatal.

\section{Método}

Trata-se da aplicação do método da Pesquisa Convergente Assistencial (PCA) (Trentini et al., 2017) para a construção de objetos de aprendizagem interativos e a organização em uma Matriz de Design Instrucional (Costa et al., 2014), voltada para a intervenção na prática cotidiana de cuidados aos recém-nascidos pré-termos (RNPT), em Unidade de Terapia Intensiva Neonatal (UTIN). 
É relevante mencionar que este artigo é originado, para submissão a um congresso, à partir de um produto desenvolvido em uma dissertação de mestrado, na modalidade profissional, as atividades foram ajustadas para operacionalização remota em virtude do advento do cenário provocado pela COVID-19.

O conceito de convergência na PCA é entendido como sendo o entrecruzamento entre as ações de assistência com as ações de pesquisa, encontro este que proporciona possibilidades de leitura e descoberta de novos fenômenos. Constituem-se atributos essenciais da PCA (Trentini et al., 2017): imersibilidade; simultaneidade; expansibilidade; e, dialogicidade. Ainda, como fonte de transformação não imediata, busca a construção de conhecimento atribuídos à prática para resolução de problemas. A PCA deve ser desenvolvida considerando-se 4 Fases: $1^{\mathrm{a}}$ ) de Concepção; $2^{\mathrm{a}}$ ) de Instrumentalização; $3^{a}$ )Perscrutação; 4르) Avaliação. (Trentini et al., 2014). As Oficinas constituíram-se veículos para a perscrutação, na qual o autor busca estratégias para levantar dados, mediante os registros, com objetivo de investigação (Trentini et al., 2014).

A intervenção, que abrangeu todas as etapas propostas pela PCA, envolveu a equipe multiprofissional que atua diretamente no cuidado de prematuros, em UTIN de um grande hospital público e de ensino, localizado na capital do estado do Paraná, Brasil. Instituição é referência para o atendimento de alto risco materno e infantil e, recebeu em 2014, do Ministério da Saúde, a certificação de Centro de Referência Estadual para Atenção Humanizada ao Recém-Nascido, o Método Canguru (Rede Neonatal, 2018). A UTIN conta com 126 profissionais de saúde dos quais foram alcançados 68 (cerca de 54\%), na primeira abordagem em relato. A capacidade de atendimento é disponibilizada por 10 (dez) leitos de UTIN, 15 (quinze) leitos para Unidades de Cuidado Intermediário Neonatal Convencional (UCINCo) e 5 (cinco) leitos para Unidade de Cuidado Intermediário Neonatal Canguru (UCINCa), caracterizando-se como UTIN Tipo III, conforme Portaria no 930, de 10 de maio, de 2012 (Ministério da Saúde [MS], 2012).

Para seleção dos interessados, adotaram-se os critérios de inclusão: ser profissional de saúde da UTIN do hospital em foco, independente da área formação; e, prestar cuidado direto ao RNPT. Não houve critério de exclusão, mesmo os afastados (saúde ou férias) puderam participar devido o processo ter sido remoto. A abordagem pedagógica interativa foi desenvolvida a partir de um projeto pedagógico, com cronograma das oficinas desenvolvidas em um Ambiente Virtual de Aprendizagem (AVA), com apoio da Plataforma Moodle. Em fevereiro de 2020, o planejamento foi apresentado para análise e parecer do Centro de Estudos e Pesquisa em Enfermagem (CEPEn), setor responsável pelas capacitações na instituição. Frente a aprovação, foi implementado o programa que se desenvolveu com nove oficinas instrucionais, os profissionais foram orientados a cumprir com todas as oficinas no período entre 26 de junho a 12 agosto de 2020 , cerca de 50 dias, considerando que estavam trabalhando normalmente e deveriam se organizar. A carga horária total das Oficinas foi de $20 \mathrm{~h}$ e, os participantes receberam certificados.

A duração de cada oficina foi cerca de uma hora e, incluíram-se as atividades de: frequência às videoaula; resposta à pergunta disparadora registrada na plataforma; leitura dos artigos científicos disponibilizados; apreciação dos vídeos sugeridos como complementares; e, a participação em grupo de discussão, foi instituído um fórum virtual. Cada oficina apresentou um tema singular para reflexão do ambiente de trabalho, inserindo as práticas que buscam a garantia do cuidado, qualificado e seguro, para o desenvolvimento do RNPT. E, os dados foram coletados mediante os registros no fórum virtual, consolidando-se como produtos de discussão do grupo organizados de acordo com os temas abordados, sem codificação, como estratégia de coleta de dados, na Fase da Perscrutação da PCA.

A organização das oficinas no processo de ensino-aprendizagem orientou-se por Afonso (2013), como um encontro de indivíduos com o objetivo de promover o trabalho em grupo. No presente, foram encontros remotos, não síncronos, para a promoção de um trabalho coletivo de produção de uma tecnologia assistencial, à partir da Matriz de Design Instrucional. 
Para Afonso, uma oficina em saúde, devido sua relevância, deve ser aceita pelo grupo e seu desenvolvimento ocorrer em quatro etapas: 1 . Demanda: aborda as necessidades a serem trabalhadas podendo, ao longo do processo, se diferenciar da proposta inicial. 2. Pré-análise: inclui o levantamento de dados e aspectos importantes da questão a ser trabalhada. 3. Foco e enquadre: o foco se refere ao trabalho que será desenvolvido, já o enquadre inclui o número e tipo de participantes, o contexto institucional, o local, os recursos disponíveis e o número de encontros. 4. Planejamento flexível: define como o coordenador da tarefa deve planejar cada encontro.

Ressalta-se ainda que as Oficinas se inserem na Política Nacional de Educação Permanente em Saúde (PNEPS) como uma proposta de aprendizagem no trabalho, incorporada ao cotidiano das instituições para a transformação das práticas profissionais (Ministério da Saúde [MS], 2018).

O projeto foi aprovado pelo Comitê de Ética de Pesquisa em Seres Humanos, conforme previsto na Resolução no 466/12 do Conselho Nacional de Saúde (Torres, Mendes \& de Souza, 2019), sob CAAEE n²5514119.7.0000.0096.

\section{Resultados}

A construção das Oficinas visaram sobretudo a interatividade e inclusão multiprofissional e, para cada uma seguiram-se as etapas propostas por Afonso (2013) objetivando a construção das temáticas e o cronograma da Matriz de Design Instrucional (MDI), ressaltando-se que não necessariamente foram desenvolvidas em ordem sequencial. São apresentadas neste capítulo as Etapas para elaboração de cada Oficina e, na Discussão são descritas cada Oficina, com a sustentação teórica.

Na 1aㅡ Etapa, a de Demanda, direcionou-se uma pergunta disparadora, a saber: Como podemos melhorar a nossa prática? E, com esta, foi abordada a fisiologia do desenvolvimento cerebral do feto, seu processo de maturação, o desenvolvimento do aparelho psíquico-emocional, correlacionaram-se as intercorrências ao desenvolvimento do prematuro e, enfatizada a importância de um planejamento assistencial institucional.

$\mathrm{Na} 2^{a}$ Etapa, de Pré-análise, os objetivos de cada oficina foram traçados, assim como definidos os conteúdos teóricos necessários para cada abordagem e, a organização didática da gravação das videoaulas para disponibilização na plataforma Moodle. A temática foi baseada no desenvolvimento prévio de uma Scoping Review (ScR), valendose do acrônimo PCC, a saber: Quais os cuidados desenvolvimentais mais evidenciados aos neonatos prematuros assistidos em Unidade de Terapia Intensiva Neonatal? Nesta revisão de escopo os artigos selecionados abordaram as seguintes categorias de cuidados: NIDCAP; uso de sacarose durante procedimentos dolorosos; Método Canguru; a música; protocolo de cuidado para exame de retinopatia; a voz materna; o desenvolvimento de protocolos de cuidado; e, o banho Swaddle.

A produção das videoaulas foi solicitada aos profissionais da própria instituição hospitalar e do cenário de cuidado em estudo, sobretudo os líderes das equipes assistenciais e dos turnos, que detinham expertise sobre cada enfoque a ser desenvolvido, nas nove Oficinas; e, ainda profissionais em curso de pós-graduação Stricto Sensu. Foram eles: médico neonatologista; enfermeiros; fisioterapeutas; fonoaudióloga; psicólogo e assistente social; e, uma enfermeira em programa de doutoramento em enfermagem. Ressalta-se que os profissionais receberam orientação quanto ao escopo das videoaulas e da MDI, para procederem à gravação.

Foi estabelecida assim uma MDI, intitulada "Cuidar do Prematuro é Preservar o seu Cérebro em Desenvolvimento", com criação de um logotipo que marcou todo o material, nele consta uma imagem que expressa o cuidado ao neonato preservando o seu desenvolvimento neurológico (Fig. 1). 


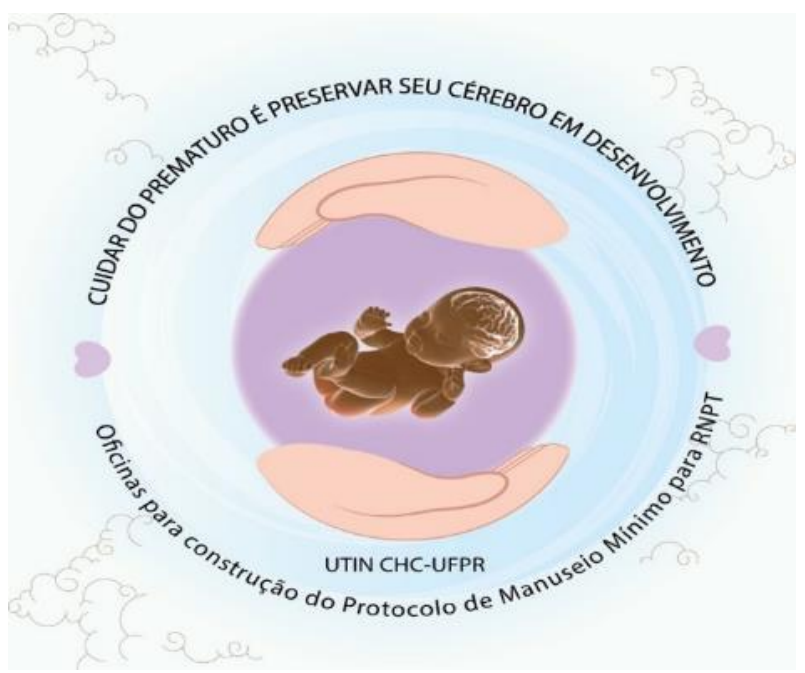

Fig. 1. Logotipo do material da Matriz de Design Instrucional. Curitiba, Brasil, 2020. Fonte: As autoras (2020).

Os objetos de aprendizagem interativos como os temas, foram operacionalizados com oficinas remotas assíncronas, focados na abordagem e estímulos para a discussão de questões centrais, aplicáveis à construção de novas práticas na área de saúde, mais especificamente no cuidado ao RNPT (Knak \& Paz, 2016).

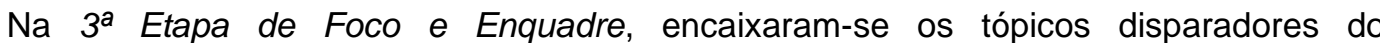
desenvolvimento das oficinas, catalisando a reflexão da realidade do ambiente de trabalho e das possibilidades de mudanças das práticas assistenciais.

Por fim, como 4aㅡ Etapa, a do Planejamento flexível, ocorreram as Oficinas com reflexões provocadas por perguntas disparadoras, para as quais se receberam sugestões pelos profissionais participantes. Sendo que todas as sugestões foram lidas atentamente pela pesquisadora principal, para a realização da seleção e categorização dos relatos pertinentes aos temas e, relevantes para construção de uma tecnologia educacional., propósito maior do desenvolvimento da dissertação de mestrado.

As oficinas foram desenvolvidas em nove módulos, sendo oito tópicos com abordagens teórico-científicas e práticas específicas e, o nono dedicado ao fechamento e produção da tecnologia educacional. Foram as seguintes: Oficina I: abordagem da fisiologia do desenvolvimento cerebral do feto; Oficina II: análise do grupo dos contextos de vulnerabilidade familiar e seus respectivos fatores; Oficina III: reflexão dos desafios relacionados ao aleitamento materno do RNPT; Oficina IV: abordagem da fisioterapia aplicada aos cuidados neurodesenvolvimentais do prematuro; Oficina $V$ : a importância da estabilidade térmica do RNPT; Oficina VI: princípios e fundamentos do cuidado desenvolvimental; Oficina VII: processo fisiológico da dor no recém-nascido prematuro, seus indicadores e suas consequências quando negligenciada; Oficina VIII: reconhecimento e avaliação da dor por meio de escalas validadas; Oficina IX: Análise das sugestões e dos produtos do chat (resultado da discussão do grupo), das respostas à pergunta disparadora, para o desenvolvimento do protocolo de manuseio mínimo ao recém-nascido em cuidados intensivos.

Contou-se com a participação de 68 profissionais da equipe multidisciplinar, contabilizando cerca de $54 \%$ de toda a equipe de profissionais atuantes na unidade responsável pela assistência direta aos prematuros no cenário da pesquisa, sendo: $31(45,6 \%)$ técnicos/as de enfermagem; 25 (36,8\%) enfermeiros/as; 5 (7,3\%) médicos neonatologistas; 4 (5,9\%) fisioterapeutas; 1 (1,5\%) auxiliar de enfermagem; 1 (1,5\%) fonoaudióloga; e, 1 (1,5\%) profissional que era Assistente Social e Psicóloga, exercendo duas funções em regime de contratação distintos mas, coexistentes na instituição. 
A Oficina IX, foi planejada e organizada para categorização e desenvolvimento da tecnologia assistencial propriamente dita, ao término das oficinas educativas, valendo-se da ferramenta Wikiß de compartilhamento de informações em material online dentro da plataforma das oficinas. Após a estruturação da primeira versão do documento este foi disponibilizado na plataforma Moodle, como prévia dos principais cuidados desenvolvimentais descritos na literatura e, posteriormente, foi editado pelo grupo.

Em síntese, cada Oficina estabeleceu-se em dois ciclos entrelaçados com o objetivo de promover a difusão e o compartilhamento dos conhecimentos, científicos, pessoais e empíricos: $1^{\circ}$ ) difusão das informações; e $2^{\circ}$ ) incentivo ao debate e discussão da temática. Assim, os participantes puderam contribuir com a triangulação de informações procedentes das videoaulas - pergunta-disparadora - chat - reflexão -sugestões.

Ao final do processo das Oficinas foi disponibilizado um instrumento de avaliação sobre a abordagem utilizada e seu impacto, obteve-se retorno de $52(76,47 \%)$ cursistas, os quais consideraram-na relevante para qualificação da prática assistencial na unidade pois, possibilitou a releitura da prática e agregação de conhecimentos científicos.

\section{Discussão}

$\mathrm{Na}$ Oficina / com a abordagem ao desenvolvimento cerebral fetal, a importância do cuidado protetor ao recém-nascido, e a necessidade crucial do cuidado com o desenvolvimento, foram apresentadas estratégias de prevenção da hemorragia intracraniana periintraventricular. Estas foram descritas pelas autoras Knak e Paz (2016) como o posicionamento mediano do prematuro, a diminuição de estressores ambientais - como a manipulação mínima com agrupamento de cuidados - e, o controle térmico. O grupo fol estimulado a refletir sobre a identificação das consequências da prematuridade no desenvolvimento do RNPT, de que maneira poder-se-ia ajustar o foco assistencial aos prematuros na sua unidade, às recomendações. Neste sentido, pesquisadores enfatizam a importância do planejamento institucional na UTIN, como o ambiente que acolhe o RNPT após o nascimento mas, que também exerce impactos deletérios ao seu organismo (Magalhães et al., 2011), que podem comprometer o desenvolvimento por toda a vida (Rabello \& Barros, 2011; Sousa et al., 2008).

A Oficina II, com foco na estratégia do acolhimento familiar para a promoção do vínculo na Unidade de Terapia Intensiva, instigou o profissional para análise dos contextos de vulnerabilidade familiar e seus respectivos fatores e, apontou para a importância do reconhecimento de seu papel na promoção do acolhimento familiar. O grupo de discussão abordou metas de melhorias para promoção do acolhimento familiar e, posteriormente, foram descritas no chat as atitudes exitosas, presenciadas na unidade. Sabe-se que o ambiente não atua como estressor apenas para o RNPT, mas também para seus familiares, devido ser estranho e impessoal (Del'Angelo et al., 2010). Sendo assim, o cuidado prestado pelo enfermeiro deve ser estendido aos familiares, através de ações que incentivem a sua permanência ao lado do paciente, que promovam conforto, esclareçam dúvidas, incentivando uma assistência individualizada que atenda ao interesse de todos (Guimarães \& Monticelli, 2007). Os pais podem apresentar problemas emocionais nesse período, em decorrência do medo ao enfrentamento das dificuldades e a fragilidade para a construção do apego (Cunha et al., 2020).

Atentando para a importância do aporte nutricional, desde o início do internamento do prematuro, e de suas dificuldades, a Oficina III, disponibilizou a reflexão e materiais relacionados aos desafios do aleitamento materno ao RNPT, estimulando a compreensão do processo anatômico e fisiológico relacionado à sucção nutritiva ou não. Lembrando ainda que, o domínio dos métodos de estímulo ao aleitamento materno do prematuro é indicativo da qualidade assistencial e, interfere positivamente no processo de oferta diária na unidade. Segundo pesquisadores, o tempo de internamento interfere negativamente no processo do aleitamento materno e, o gerenciamento dos momentos de ordenhas acabam sendo prejudicados durante as diárias estressantes das UTIN (Rolim et al., 2012). 
A Oficina IV foi desenvolvida com foco nos cuidados neurodesenvolvimentais do prematuro, como: aspiração segura; contenção facilitada; a importância do ninho para a posturação do RNPT; e, em especial sobre o posicionamento nas primeiras 72 horas de vida para a prevenção da hemorragia cerebral - esta como a lesão mais relevante no período neonatal-, devido à predisposição anatômica. De acordo com Knak \& Paz (2016), o posicionamento mediano da cabeça em posição dorsal ou lateral, contribui para um fluxo cerebral de forma homogênea e contínua, evitando rupturas de capilares fragilizados.

A Oficina $V$ abordou a importância da estabilidade térmica do RNPT, mostrando a fisiologia de perda e produção de calor do prematuro. Foi apresentando complementarmente, um relato do processo de construção de um protocolo de umidificação, já implementado na unidade em estudo. Os participantes foram incentivados à identificação de estratégias para manutenção do controle térmico. Sabe-se que a termorregulação é uma função de controle de temperatura corpórea intimamente relacionada com a transição e sobrevivência dos neonatos. A equipe deve estar atenta e apta para intervir sempre que houver alguma alteração térmica no RNPT, identificando sinais clínicos de hipotermia e hipertermia, participando do plano de cuidados, avaliando o conhecimento e as técnicas em busca de melhores resultados e, por fim, prevenindo a morbimortalidade no período neonatal (Ministério da Saúde [MS], 2014). A exposição a procedimentos prolongados e a não sistematização do cuidado eleva as aberturas das portinholas, gerando hipotermias. A redução da temperatura corporal gera diminuição da produção de surfactante e, aumento do consumo de oxigênio, culminando com o desenvolvimento ou a piora da insuficiência respiratória (Harillo Aceved, Ricco Becerra, \& López Martíne, 2017).

Considerando a seriedade de cuidar do RNPT com foco no desenvolvimento futuro, a Oficina VI promoveu uma analogia acerca dos princípios e fundamentos do cuidado desenvolvimental, remetendo o grupo para o manuseio mínimo e, ao processo da construção da MDI. Ao final, foi solicitado aos profissionais que descrevessem quais os cuidados desenvolvimentais - que protegem o desenvolvimento neurológico do RNPT poderiam ser implantados para o fortalecimento de uma tecnologia assistencial. A implementação do NIDCAP nas UTIN pode proporcionar efeitos positivos no crescimento e recuperação do RNPT, de modo a preconizar ações de acordo com a precisão clínica e desenvolvimental, minimizar o manuseio excessivo do bebê, adaptar o ambiente para proporcionar mais conforto e instituir medidas para inserir os pais no cuidado (Mirlashari et al., 2019). Entre os benefícios do NIDCAP, destaca-se o modelo específico a essa população descrita e, principalmente em proporcionar a segurança aos cuidadores na assistência ao prematuro (Andrade, 2019).

$\mathrm{Na}$ Oficina VII foi abordado o processo fisiológico da dor, seus indicadores e suas consequências, sobretudo quando negligenciada; foram descritos os principais fármacos utilizados na neonatologia, as principais escalas para avaliação do nível de dor e estresse, além abordagem às consequências no desenvolvimento do prematuro. Conforme Silva et al (2007), o "sentir a dor" está estabelecido por sensores corporais por volta da $20^{a}$ semana de gestação, com marcadores de estresse bem estabelecidos por volta da $30^{a}$ semana de gestação. No RNPT, a dor pode ser definida como uma experiência subjetiva que pode estar relacionada a lesões reais. Contudo, não são expressadas pela verbalização, com descrição ou queixa da dor sentida, o que repercute na dificuldade da identificação e localização da mesma (Moraes, 2017), na ausência de tempos/horários e escalas para aferi-la como o quinto sinal vital. Neste sentido, a construção de protocolos assistenciais, para diagnóstico e intervenção em processos álgicos, norteia e padroniza cuidados e meios avaliativos para a tomada de condutas clínicas para minimizá-la (Melo, 2010).

Devido às iatrogênias medicamentosas ao desenvolvimento cerebral do prematuro, em primeira escolha as ações de controle da dor são voltadas para os métodos nãofarmacológicos. De maneira continuada, a Oficina VIII dispôs os conceitos para o reconhecimento e avaliação da dor, valendo-se de escalas validadas e, instigou os profissionais à reflexão das estratégias para prevenção e redução da dor no RNPT. As escalas são ferramentas relevantes de informações para identificação da dor e, podem ser utilizadas em prematuros, a Neonatal Infant Pain Scale (NIPS) - a Escala de Avaliação de Dor Neonatal-, é utilizada na unidade da pesquisa. 
Revisando todo o processo das oficinas anteriores, a Oficina $I X$ foi destinada para a sistematização e a construção do protocolo assistencial, através da plataforma Wiki® com adesão às contribuições do grupo em cada categoria de cuidado, a saber: ambiente; cuidados de rotina; procedimentos terapêuticos; procedimentos de monitorização; e cuidado compartilhado familiar.

\section{Considerações Finais}

Considera-se respondida a pergunta de pesquisa e, alcançado o objetivo traçado para esta apresentação, foi descrito parte do processo de uma Pesquisa Convergente Assistencial, a qual foi ancorada pela Matriz de Design Instrucional, visando a intervenção nos cuidados aos recém-nascidos pré-termos em UTIN.

Este estudo teve como "limitação" a adaptação do modo presencial para o remoto, operacionalizada em curto espaço de tempo, devido às novas diretrizes institucionais necessárias ao cenário da pandemia do Covid-19. Contudo, este ajuste favoreceu a participação, inclusive, dos profissionais afastados temporariamente, por qualquer motivo, do serviço, podendo-se interpretá-lo como um processo interativo inclusivo.

Destaca-se que a Pesquisa Convergente Assistencial baseada em uma Matriz de Design Instrucional compreendendo as oficinas virtuais assíncronas, planejadas e sustentadas teoricamente, cumpriu com a difusão de informações científicas de maneira prática, possibilitando o ágil acesso do profissional ao conteúdo específico para o desenvolvimento da temática. Houve translação e atualização do conhecimento ao profissional, instrumentalizando-o à participação na construção de um cuidado qualificado, de acordo com as propostas instituídas nas videolaulas e nos debates, além de ter sido preservada a possibilidade de esclarecimento de dúvidas.

E, para além desta etapa de pesquisa apresentada, culminou-se com o desenvolvimento de um Protocolo de Manuseio Mínimo para Recém-nascidos Prematuros em Unidade de Terapia Intensiva Neonatal, de um grande hospital público de referência para o alto risco, desenvolvido com a participação multiprofissional da equipe atuante no cenário, o qual foi implantado e implementado. Atendendo-se assim à fragilidade de a UTIN ser um ambiente estressor e invasivo e requerer ferramentas que minimizem seus efeitos deletérios.

Neste sentido, para a sociedade contribuiu-se com a redução da morbidade, das sequelas do desenvolvimento em longo prazo e, da mortalidade dos prematuros com o avanço do conhecimento científico e técnico relevante para a qualificação assistencial.

Para as instituições de saúde, a pesquisa reafirmou a necessidade de abordagens inovadoras de educação permanente, com perspectiva de cuidado ampliada, centrada na promoção do desenvolvimento do prematuro, minimizando eventos adversos no período de internação. Acredita-se que assim, poder-se-á conquistar avanços no comportamento assistencial pela equipe multidisciplinar. Este estudo pode ser reproduzido em outros cenários, singularizando-se às necessidades emergentes.

\section{Agradecimentos}

Agradecemos à oportunidade oferecida pelos programas brasileiros de pós-graduação na modalidade profissional, de o profissional rever sua prática e nela intervir com criatividade e inovação. 


\section{Referências}

Abdeyazdan, Z., Ghasemi, S., Marofi, M., \& Berjis, N. (2014). Motor responses and weight gaining in neonates through use of two methods of earmuff and receiving silence in NICU. The Scientific World Journal, 2014, 1-5. http://dx.doi.org/10.1155/2014/864780

Afonso, M. L. M. (2013). Oficinas em dinâmica de grupo na área da saúde. (1 $\left.{ }^{a} e d.\right)$. Casa do psicólogo.

Andrade, L. M. M. (2019). Exposição e manejo da dor em recém-nascidos prematuros durante o tempo de hospitalização na Unidade de Terapia Intensiva Neonatal [Masters dissertation, Universidade de Brasília]. Repositório Institucional da UNB. https://repositorio.unb.br/bitstream/10482/37516/1/2019_LaryssaMarinnaMadeiradeAndrade. pdf

Costa, I. A., Kemczinski, A., Gasparini, I., \& Souza, D. C. d. (2014). Matriz de design instrucional da metodologia para a construção de objetos de aprendizagem interativos. Nuevas Ideas en Informática Educativa TISE. http://www.tise.cl/volumen10/TISE2014/tise2014_submission_307.pdf

Cunha, G. M. d., Rodrigues, F. A., \& Herber, S. (2020). Aleitamento materno do prematuro em um hospital amigo da criança. Revista Recien, 10(30), 168-178.

Del'Angelo, N., de Goes Fdos, S., Dalri, M., Leite, A. M., Furtado, M., \& Scochi, C. (2010). Nursing diagnoses of premature infant under intermediary care. Revista Brasileira de Enfermagem, 63(5), 755-761.

Gaspardo, C. M., Martinez, F. E., \& Linhares, M. B. M. (2010). Cuidado ao desenvolvimento: intervenções de proteção ao desenvolvimento inicial de recém-nascidos pré-termo. Revista Paulista de Pediatria, 28(1), 77-85.

Guimarães, G. P., \& Monticelli, M. (2007). (Des) motivação da puérpera para praticar o método mãe-canguru. Revista Gaúcha de Enfermagem, 28(1), 11.

Harillo Acevedo, D., Rico Becerra, J. I., \& López Martínez, Á. (2017). La filosofía de los cuidados centrados en el desarrollo del recién nacido prematuro (NIDCAP): una revisión de la literatura. Enfermería Global, 16(48), 577-589.

Knak, D., \& Paz, I. (2016). Cuidando do cerebro em desenvolvimento: estratégias de prevenção de hemorragia intraccraniana peri-intraventricularem UTI neonatal. Anais do Salão de Ensino e de Extensão, 144.

https://online.unisc.br/acadnet/anais/index.php/salao_ensino_extensao/article/view/15175

Magalhães, L. d. C., Fonseca, K., Martins, L., \& Dornelas, L. d. F. (2011). The performance of preterm children with very and extreme low weight according to the Denver-II test. Revista Brasileira de Saúde Materno Infantil, 11(4), 445-453.

Marski, B. d. S. L., Facio, B. C., Ichisato, S. M. T., Barba, P. C. d. S. D., \& Wernet, M. (2018). Developmental Care: assistance of nurses from Neonatal Intensive Care Units. Revista Brasileira de Enfermagem, 71, 2758-2766.

Ministério da Saúde. (2018). Política Nacional de Educação Permanente em Saúde: o que se tem produzido para o seu fortalecimento?

https://bvsms.saude.gov.br/bvs/publicacoes/politica_nacional_educacao_permanente_saude fortalecimento.pdf

Ministério da Saúde. (2014). Atenção à saúde do recém-nascido: guia para os profissionais da saúde. http://bvsms.saude.gov.br/bvs/publicacoes/atencao_saude_recem_nascido_v1.pdf

Ministério da Saúde. (2012). Portaria no 930, de 10 de maio de 2012. http://bvsms.saude.gov.br/bvs/saudelegis/gm/2012/prt0930_10_05_2012.html

Mirlashari, J., Fomani, F. K., Brown, H., \& Tabarsy, B. (2019). Nurses' and Physicians' Experiences of the NIDCAP Model Implementation in Neonatal Intensive Care Units in Iran. Journal of pediatric nursing, 45, e79-e88.

Moraes, E. L. L. d. (2017). Protocolo multiprofissional para manejo da dor e do estresse em recém-nascidos: uma pesquisa-ação [Martes's dissertation, Universidade Federal do Paraná]. Biblioteca digital. https://acervodigital.ufpr.br/handle/1884/53384 
Rabello, M. S. d. C., \& Barros, S. M. O. d. (2011). Clinical and epidemiological aspects of prematurity in a Normal Delivery Center, São Paulo, Brazil. Einstein, 9(4), 483-488.

Rede Neonatal. (2012). Institucional. http://www.redeneonatal.com.br/index.php/institucional.

Rolim, K. M. C., Barbosa, R. d. M. A., Medeiros, R. M. G., Leite, M. L., \& Gurgel, E. d. P. P. (2012). Permanence of the semipermeable membrane in the newborn skin: a differentiated care. Revista Rene, 11(1).

Sousa, M., Silva, R., \& Araújo, S. (2008). Quantification of manipulations in neonatal intensive care unit: proposal of protocol elaboration. ConScient Saúde, 7(2), 269-274.

Torres, J., Mendes, A., \& de Souza, M. V. (2019). Objetos de aprendizagem: uma proposta interativa de desenvolvimento. Brazilian Journal of Development, 5(11), 23377-23398.

Trentini, M., Paim, L., \& Silva, D. (2014). Pesquisa convergente assistencial: delineamento provocador de mudanças nas práticas de saúde. Moriá Editora.

Trentini, M., Paim, L., \& Silva, D. M. G. V. (2017). O método da pesquisa convergente assistencial e sua aplicação na prática de enfermagem. Texto \& Contexto-Enfermagem, 26(4).

Organização das Nações Unidas (2019). OMS: cerca de 30 milhões de bebês nascem prematuros por ano no mundo. https://nacoesunidas.org/oms-cerca-de-30-milhoes-de-bebes-nascemprematuros-por-ano-no-mundo/ 